\title{
The Demographics of Canine Hip Dysplasia in the United States and Canada
}

\author{
Randall T. Loder ${ }^{1}$ and Rory J. Todhunter ${ }^{2}$ \\ ${ }^{1}$ Department of Orthopaedic Surgery, Indiana University School of Medicine and James Whitcomb Riley Children's Hospital, \\ Indianapolis, IN 46202, USA \\ ${ }^{2}$ Department of Clinical Sciences, College of Veterinary Medicine, Cornell University, Ithaca, NY 14853-6401, USA \\ Correspondence should be addressed to Randall T. Loder; rloder@iupui.edu
}

Received 28 December 2016; Accepted 27 February 2017; Published 12 March 2017

Academic Editor: Antonio Ortega-Pacheco

Copyright (C) 2017 Randall T. Loder and Rory J. Todhunter. This is an open access article distributed under the Creative Commons Attribution License, which permits unrestricted use, distribution, and reproduction in any medium, provided the original work is properly cited.

\begin{abstract}
Canine hip dysplasia (CHD) is a common problem in veterinary medicine. We report the demographics of CHD using the entire hip dysplasia registry from the Orthopedic Foundation for Animals, analyzing differences by breed, sex, laterality, seasonal variation in birth, and latitude. There were 921,046 unique records. Each dog was classified using the American Kennel Club (AKC) and Fédération Cynologique Internationale (FCI) systems. Statistical analysis was performed with bivariate and logistic regression procedures. The overall CHD prevalence was $15.56 \%$. The OR for CHD was higher in females (1.05), those born in spring (1.14) and winter (1.13), and those in more southern latitudes (OR 2.12). Within AKC groups, working dogs had the highest risk of CHD (OR 1.882) with hounds being the reference group. Within FCI groups, the pinscher/molossoid group had the highest risk of CHD (OR 4.168) with sighthounds being the reference group. The similarities between CHD and DDH are striking. Within DDH there are two different types, the typical infantile DDH and the late onset adolescent/adult acetabular dysplasia, with different demographics; the demographics of CHD are more similar to the later onset DDH group. Comparative studies of both disorders should lead to a better understanding of both CHD and DDH.
\end{abstract}

\section{Introduction}

Canine hip dysplasia (CHD) is a well-known disorder in veterinary medicine [1-4], especially amongst certain breeds. The human counterpart of CHD, developmental dysplasia of the hip (DDH), is also a well-known problem with differences in prevalence by race/ethnicity [5], analogous to breed differences in CHD. Comprehensive literature reviews of $\mathrm{DDH}$ have shown various demographic patterns regarding sex, laterality, latitude, and seasonal variation in birth month $[5,6]$. Variation in birth month/season has been described in a few small series of CHD [7-12]. There has been no study of the demographics of CHD using a large data set. The purpose of this study was to investigate the demographics of CHD using a large North American data base and analyze the differences by breed, sex, laterality, seasonal variation in birth, and latitude. Comparison with the demographics of DDH may shed further light on the etiology of both conditions and specifically support the use of CHD as an animal model for $\mathrm{DDH}$, as well as DDH pointing towards further comparative research areas in CHD.

\section{Materials and Methods}

2.1. Data Source. The data for this study was the complete hip dysplasia registry (both public and private) collected by the Orthopedic Foundation for Animals (OFA) through April 2015. There were a total of 1,430,979 records. The OFA hip score uses the American Veterinary Medical Association grading system: $1=$ excellent, $2=$ good, $3=$ fair, $4=$ borderline CHD, $5=$ mild CHD, $6=$ moderate $\mathrm{CHD}$, and $7=$ severe CHD. These scores were divided into two groups: those with CHD (scores 5-7) and those without CHD (scores 1-3); the borderline score of 4 was excluded. Duplicate records, feline cases, and those with an indeterminate score were deleted. The country of origin was known in 1,130,478 dogs; the vast 
majority $(1,121,961-99.25 \%)$ were from the USA $(1,046,249)$ or Canada $(75,712)$. Dogs less than 24 or greater than 60 months of age at the time of the radiograph were next deleted, leaving 921,046 unique records which are the data for this study.

2.2. Data Groups. Each dog was classified into related breed groups using both the American Kennel Club (AKC) (http://www.akc.org) [13] and Fédération Cynologique Internationale (FCI) (http://www.fci.be/en/Nomenclature) [14] systems. Each dog was separately given an AKC and FCI group designation and analyzed separately; the two different systems were not merged. Dogs in each of these groups are relatively similar genetically $[15,16]$ and thus could be expected to respond to environmental triggers similarly, compared to dogs that do not share a common genetic background. The AKC categories are herding, hound, working, sporting, nonsporting, terrier, toy, native, hybrid, and miscellaneous groups. The FCI categories are (1) sheep and cattle dogs; (2) pinscher, schnauzer, molossoid, and Swiss mountain and Swiss cattle dogs; (3) terriers; (4) dachshunds; (5) spitz and primitive dogs; (6) scent hounds; (7) pointers; (8) retrievers, flushers, and water dogs; (9) companion and toy dogs; and (10) sighthounds.

The variables analyzed were sex, breed, season of birth, hip score, and latitude. Season of birth was arbitrarily defined as follows: winter, December through February, spring, March through May, summer, June through August, and autumn, September through November. Each state and province was grouped by latitude. The latitude where each dog was living at the time of the radiograph was placed into 4 groups defined as (1) $<30^{\circ} \mathrm{N}$, (2) $30-39^{\circ} \mathrm{N}$, (3) $40-49^{\circ} \mathrm{N}$, and $(4)>50^{\circ} \mathrm{N}$. Those $<30^{\circ} \mathrm{N}$ were Florida, Hawaii, Louisiana, Puerto Rico, Virgin Islands, and Guam. Those $30-39^{\circ} \mathrm{N}$ were Alabama, Arkansas, Arizona, California, Colorado, District of Columbia, Delaware, Georgia, Indiana, Kansas, Kentucky, Maryland, Missouri, Mississippi, North Carolina, New Mexico, Nevada, Oklahoma, South Carolina, Tennessee, Texas, Virginia, and West Virginia. Those $40-49^{\circ} \mathrm{N}$ were the states of Connecticut, Iowa, Idaho, Illinois, Maryland, Maine, Michigan, Minnesota, Montana, Nebraska, New Hampshire, New Jersey, New York, Ohio, Oregon, Pennsylvania, Rhode Island, South Dakota, Utah, Vermont, Washington, Wisconsin, and Wyoming and the provinces of New Brunswick, Newfoundland, Nova Scotia, Ontario, Prince Edward Island, and Quebec. Those $>50^{\circ} \mathrm{N}$ were the state of Alaska and the provinces of Alberta, British Columbia, Manitoba, Northwest Territories, Saskatchewan, and Yukon Territory. Although a few of the states and provinces straddle these latitude lines, each state/province was placed into the group corresponding to the major population areas.

2.3. Statistical Analysis. Demographic variables were first analyzed using bivariate analyses (Pearson's $\chi^{2}$ test) to determine differences between those with and without CHD. Next, binary multivariate logistic regression analyses were performed to determine adjusted odds ratios (OR) and 95\% [upper, lower] confidence intervals of a dog having CHD. While the American Veterinary Medical Association grading system is a numerical value, it is not a continuous variable such as the Norberg angle, but rather a categorical ordinal variable determined by subjective criteria (http://www.ofa.org/hd_grades.html - hip dysplasia, OFA Xray procedures). For this reason, $\mathrm{CHD}$ grade was considered to be a categorical variable. All statistical analyses were performed with Systat ${ }^{\mathrm{TM}} 10$ software (Chicago, IL, 2000), and $p<0.05$ was considered statistically significant.

\section{Results}

3.1. Overall Results. The hip dysplasia scores were 1 in 74,931 dogs; 2 in 601,$893 ; 3$ in 95,154; 4 in 6,$772 ; 5$ in 86,321; 6 in 47,971; and 7 in 8,004, resulting in an overall CHD prevalence of $15.56 \%$. There was significant variability in the prevalence of CHD by AKC and FCI groups, gender, latitude, and season of birth (Table 1). CHD was overall slightly more common in females, those born in spring and winter (Figure 1(a)), and those born in the more southern latitudes (Figure 1(b)). Within AKC groups, CHD was most prevalent in hybrid breeds $(21.5 \%)$ and least prevalent in hounds (10.5\%) (Figure 1(c)). Within FCI groups, it was most prevalent in group 2 (pinscher, schnauzer, molossoid, and Swiss mountain/Swiss cattle dogs) (20.4\%) and least common in group 10 (sighthounds) (5.2\%) (Figure 1(d)). Although there was a statistically significant difference in the prevalence of $\mathrm{CHD}$ by age at the time of radiography (Figure 1(e)), the variability was less than $2 \%$ and considered to not be clinically significant, especially since the oldest group of dogs had a lower prevalence of CHD than the youngest cohort. Age was thus deleted from all further analyses. There was significant variation by individual breeds. The prevalence of CHD by breeds in this study is very similar to that given on the OFA website http://www.ofa.org, even though dogs outside of Canada or the USA were excluded in our study. The complete CHD prevalence data set is given in Supplemental Table 1 in Supplementary Material available online at https://doi.org/10.1155/2017/5723476; the highest prevalence was in the bulldog $(77.7 \%)$ and the lowest in the Italian greyhound $(0.0 \%)$.

3.2. Results by Demographic Parameters. The overall OR for CHD was higher in females (1.05 [1.064, 1.039]; $\left.p<10^{-6}\right)$, those born in spring $(1.143[1.16,1.13] ; p<0.004)$, and those living in more southern latitudes $\left(<30^{\circ} \mathrm{N}\right)$ (OR 2.12; [2.21, 2.04]; $p<10^{-6}$ ). These results from the composite data set obviously reflect the proportion of breeds in the OFA database and could likely be different if the breed composition differed. Therefore, analyses for each AKC and FCI group, as well as individual breeds, were performed (Table 2). Due to small numbers in certain groups, those in the native, hybrid, and miscellaneous were excluded when analyzing by $\mathrm{AKC}$ groups and the dachshunds when analyzing by FCI groups. Within AKC groups, working dogs had the highest risk of CHD (OR 1.882) with hounds being the reference group. Within FCI groups, group 2 (pinscher, schnauzer, molossoid, and Swiss mountain/Swiss cattle dogs) had the highest risk of CHD (OR 4.168) with sighthounds being the reference group. 


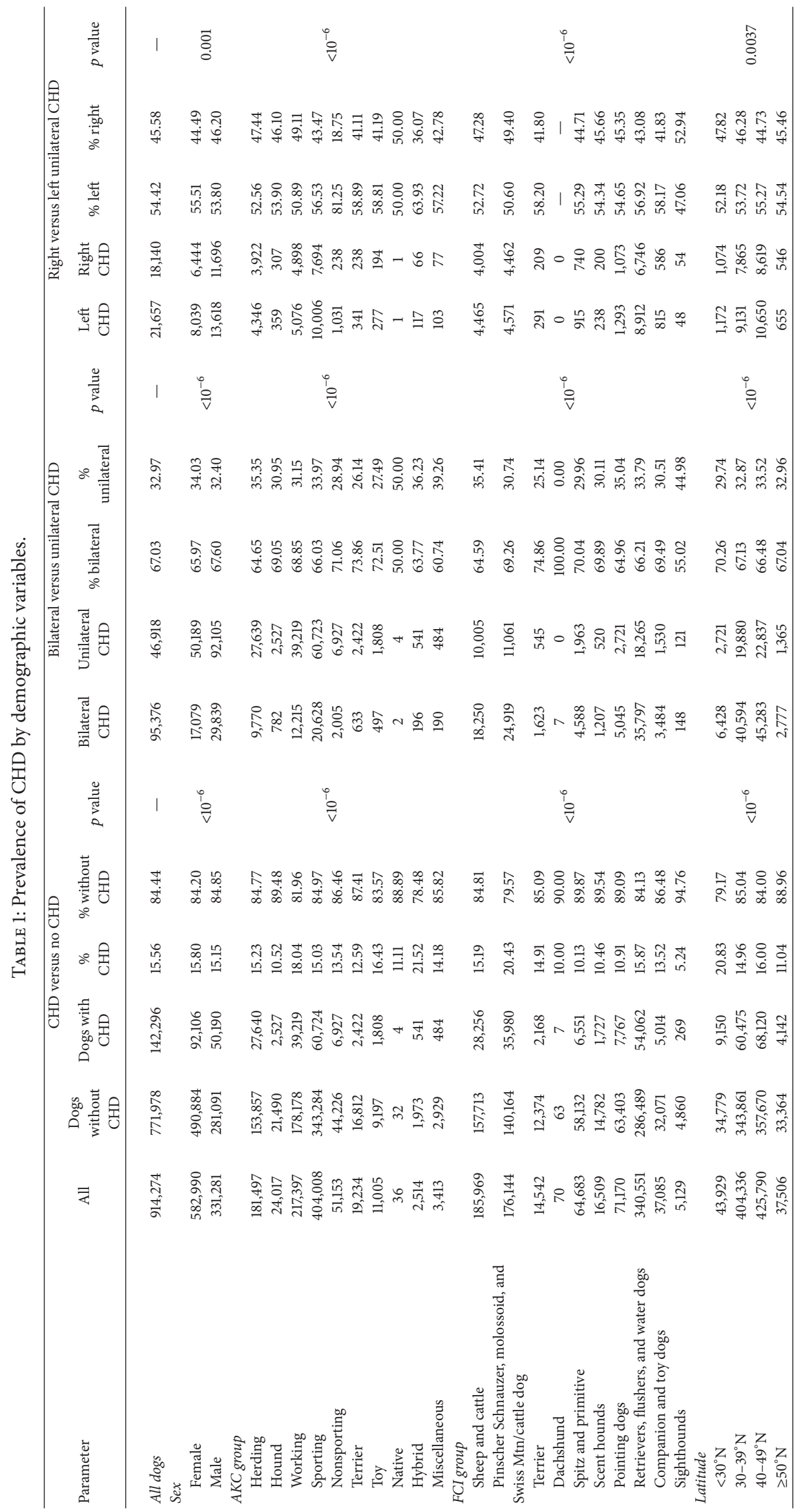




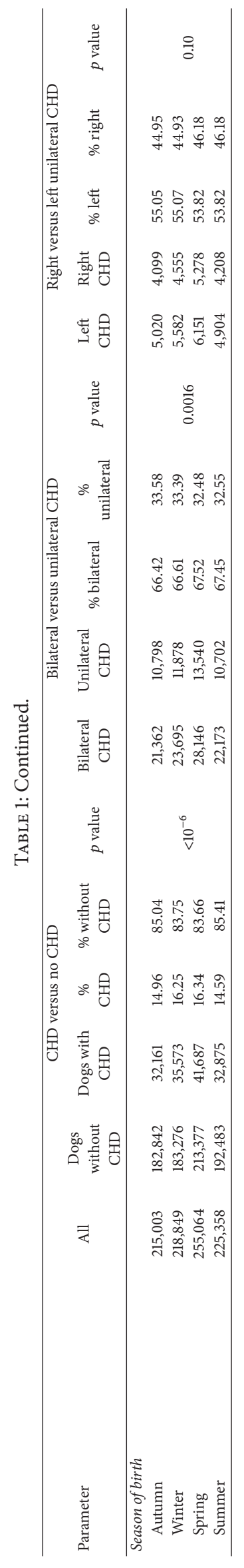




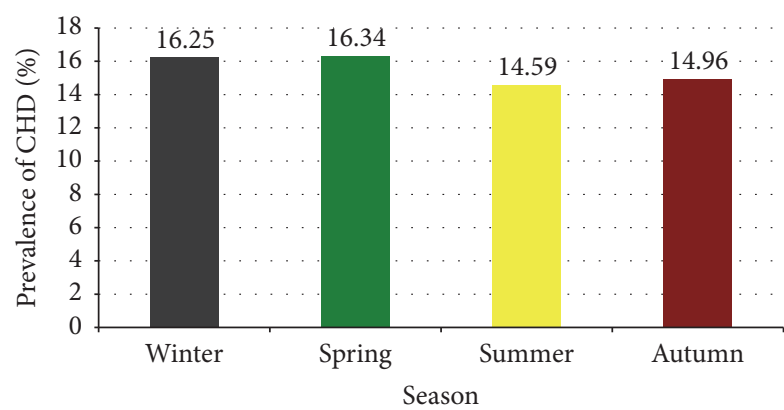

(a)

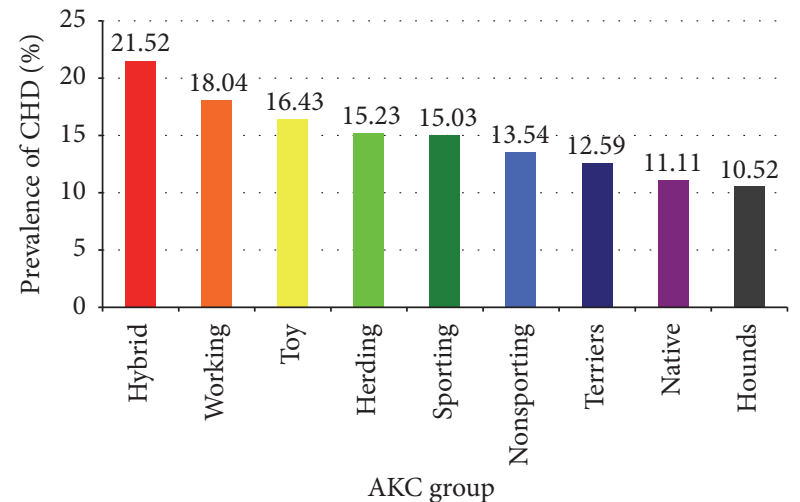

(c)

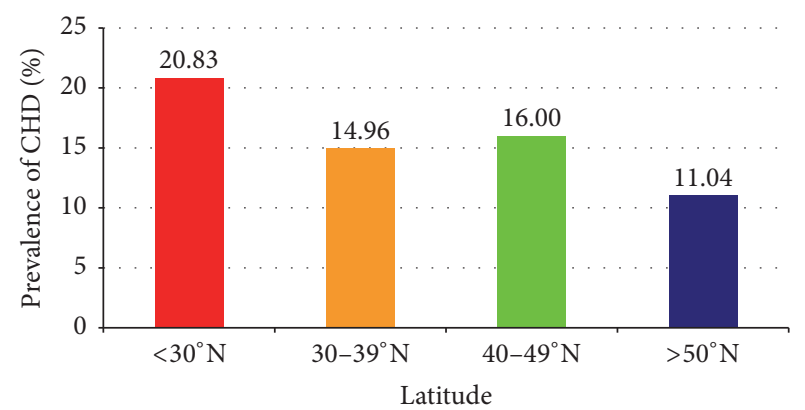

(b)

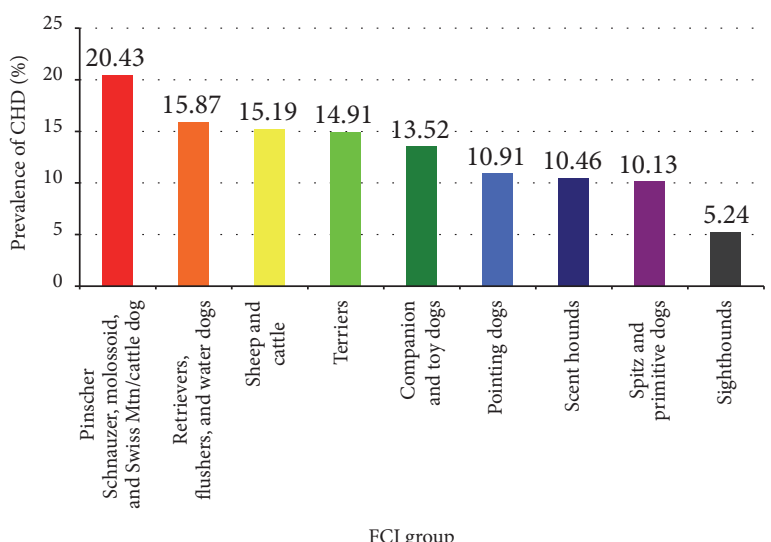

(d)

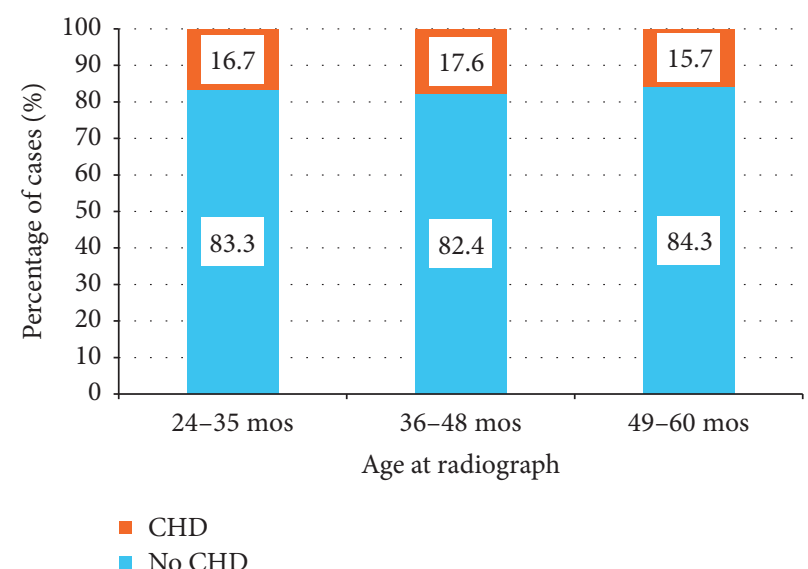

(e)

FIGURE 1: Prevalence of CHD by various demographic parameters. (a) By season of birth. (b) By latitude. (c) By AKC groups. (d) By FCI groups. (e) By age at time of radiograph. The numbers in the boxes are the percentage within each column bar.

Those born in spring had the highest risk of CHD (OR 1.14) as well as those living in latitudes $<30^{\circ} \mathrm{N}$ (OR 2.1), with a minimally higher risk in females (OR 1.05).

3.3. Results by AKC and FCI Groups. Analyses by each of the AKC and FCI groups were next performed (Table 3). Again, many of the groups showed an increase in CHD in those living in latitudes $<30^{\circ} \mathrm{N}$, except for toy dogs (where the opposite was noted with a higher risk in the most northern latitudes $>50^{\circ} \mathrm{N}$ ); hounds had no variation in CHD by latitude. When there was an increased OR by season of birth, winter and spring seasons most commonly demonstrated the increased risk with a few demonstrating an autumn increase; no group demonstrated a summer increase. A few groups demonstrated an increased CHD risk in females (AKC herding, working and sporting groups and FCI sheep/cattle and pinscher groups); sighthounds had an increased risk in male dogs.

Analyses within subgroups of AKC and FCI groups (Supplemental Table 2) as well as the most common 25 breeds 
TABLE 2: Odds ratios of CHD by AKC/FCI groups, sex, season of birth, and latitude.

(a)

\begin{tabular}{|c|c|c|c|}
\hline \multicolumn{4}{|c|}{ By AKC group } \\
\hline & OR & $95 \%$ CI & $p$ value \\
\hline \multicolumn{4}{|l|}{ Sex } \\
\hline Female & 1.056 & $(1.069,1.044)$ & $<10^{-6}$ \\
\hline Male & $1.0 \mathrm{R}$ & - & - \\
\hline \multicolumn{4}{|l|}{ Season of birth } \\
\hline Autumn & 1.025 & $(1.042,1.008)$ & $<10^{-6}$ \\
\hline Winter & 1.131 & $(1.149,1.112)$ & 0.081 \\
\hline Spring & 1.146 & $(1.165,1.128)$ & $<10^{-6}$ \\
\hline Summer & $1.0 \mathrm{R}$ & - & - \\
\hline \multicolumn{4}{|l|}{ Latitude } \\
\hline$<30^{\circ} \mathrm{N}$ & 2.116 & $(2.203,2.034)$ & $<10^{-6}$ \\
\hline $30-39^{\circ} \mathrm{N}$ & 1.428 & $(1.477,1.381)$ & $<10^{-6}$ \\
\hline $40-49^{\circ} \mathrm{N}$ & 1.552 & $(1.605,1.501)$ & $<10^{-6}$ \\
\hline$\geq 50^{\circ} \mathrm{N}$ & $1.0 \mathrm{R}$ & - & - \\
\hline \multicolumn{4}{|l|}{ AKC group } \\
\hline Herding & 1.535 & $(1.602,1.470)$ & $<10^{-6}$ \\
\hline Toy & 1.675 & $(1.788,1.570)$ & $<10^{-6}$ \\
\hline Working & 1.882 & $(1.965,1.804)$ & $<10^{-6}$ \\
\hline Sporting & 1.504 & $(1.569,1.442)$ & $<10^{-6}$ \\
\hline Nonsporting & 1.348 & $(1.415,1.284)$ & $<10^{-6}$ \\
\hline Terrier & 1.236 & $(1.311,1.164)$ & $<10^{-6}$ \\
\hline Hound & $1.0 \mathrm{R}$ & - & - \\
\hline
\end{tabular}

(b)

\begin{tabular}{|c|c|c|c|}
\hline \multicolumn{4}{|c|}{ By FCI group } \\
\hline & OR & $95 \% \mathrm{CI}$ & $p$ value \\
\hline \multicolumn{4}{|l|}{ Sex } \\
\hline Female & 1.053 & $(1.065,1.040)$ & $<10^{-6}$ \\
\hline Male & $1.0 \mathrm{R}$ & - & - \\
\hline \multicolumn{4}{|l|}{ Season of birth } \\
\hline Autumn & 1.021 & $(1.038,1.004)$ & 0.016 \\
\hline Winter & 1.124 & $(1.142,1.105)$ & $<10^{-6}$ \\
\hline Spring & 1.143 & $(1.161,1.125)$ & $<10^{-6}$ \\
\hline Summer & $1.0 \mathrm{R}$ & - & - \\
\hline \multicolumn{4}{|l|}{ Latitude } \\
\hline$<30^{\circ} \mathrm{N}$ & 2.047 & $(2.13,1.967)$ & $<10^{-6}$ \\
\hline $30-39^{\circ} \mathrm{N}$ & 1.410 & $(1.458,1.363)$ & $<10^{-6}$ \\
\hline $40-49^{\circ} \mathrm{N}$ & 1.546 & $(1.599,1.496)$ & $<10^{-6}$ \\
\hline$\geq 50^{\circ} \mathrm{N}$ & $1.0 \mathrm{R}$ & - & - \\
\hline \multicolumn{4}{|l|}{ FCI group } \\
\hline Sheep and cattle & 3.229 & $(3.653,2.854)$ & $<10^{-6}$ \\
\hline Pinscher schnauzer, molossoid, and Swiss Mtn/cattle dog & 4.618 & $(5.224,4.082)$ & $<10^{-6}$ \\
\hline Terrier & 3.163 & $(3.605,2.774)$ & $<10^{-6}$ \\
\hline Spitz and primitive & 2.059 & $(2.334,1.816)$ & $<10^{-6}$ \\
\hline Scent hounds & 2.096 & $(2.393,1.836)$ & $<10^{-6}$ \\
\hline Pointing dogs & 2.184 & $(2.473,1.927)$ & $<10^{-6}$ \\
\hline Retrievers, flushers, and water dogs & 3.386 & $(3.830,2.994)$ & $<10^{-6}$ \\
\hline Companion and toy dogs & 2.824 & $(3.204,2.489)$ & $<10^{-6}$ \\
\hline Sighthounds & $1.0 \mathrm{R}$ & - & - \\
\hline
\end{tabular}




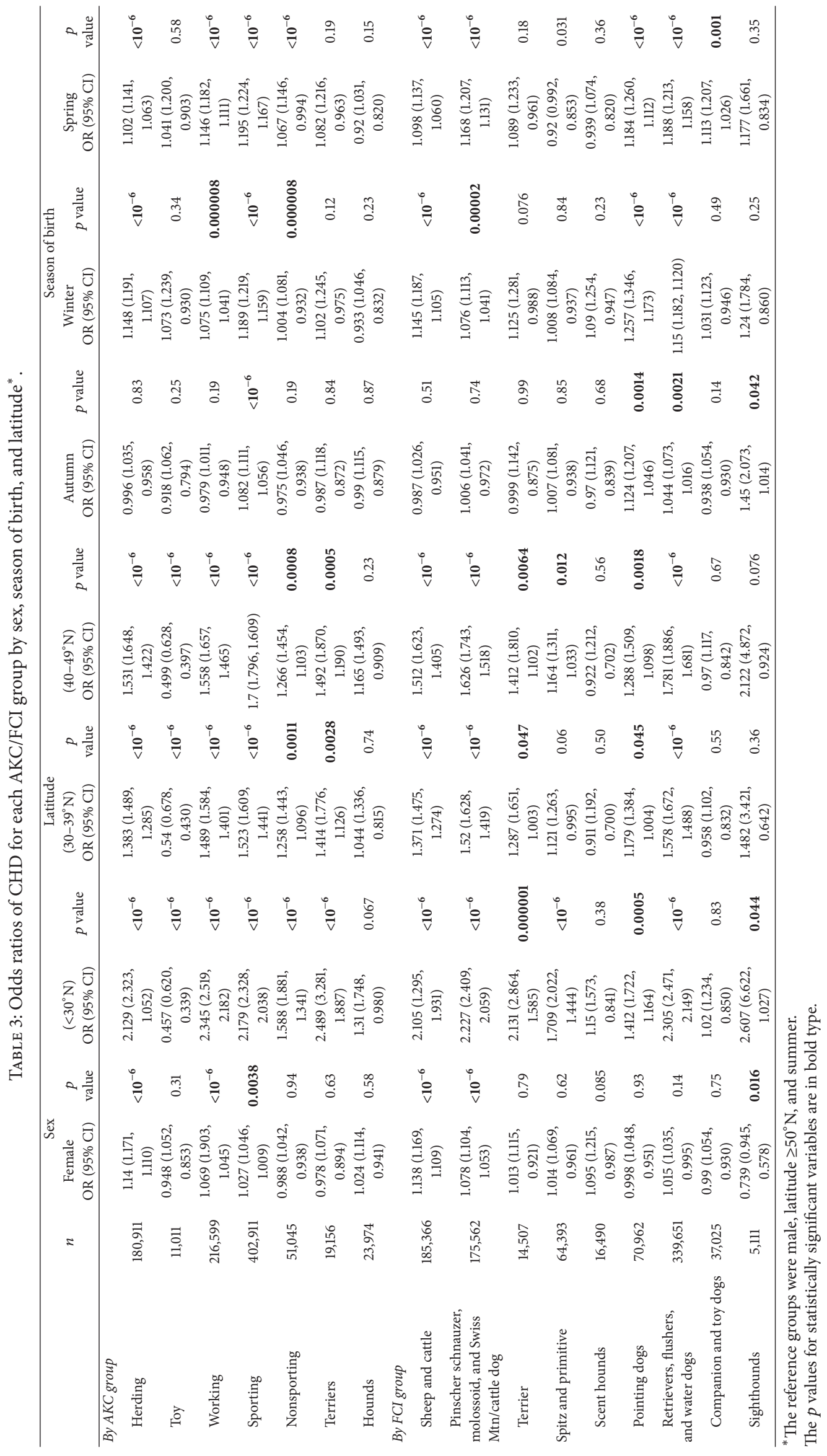




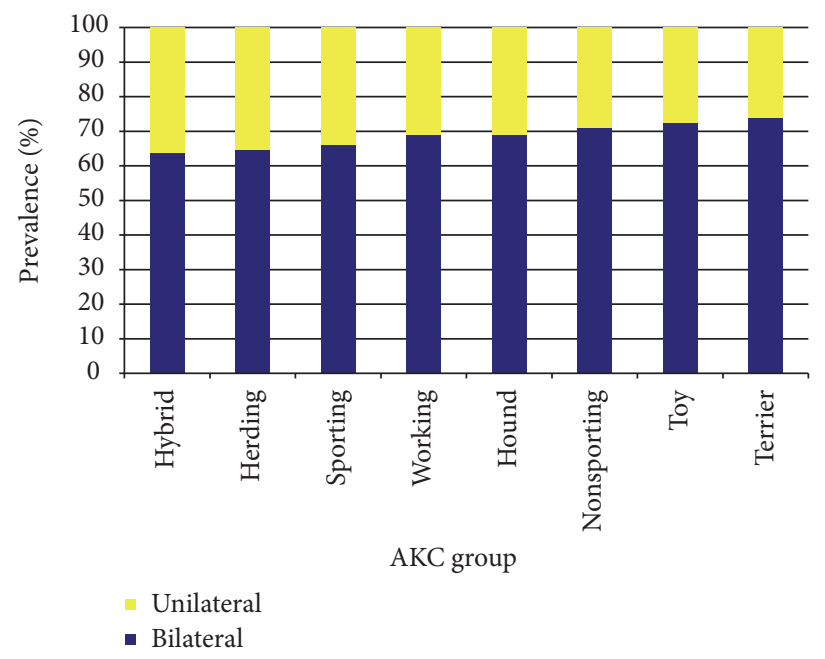

(a)

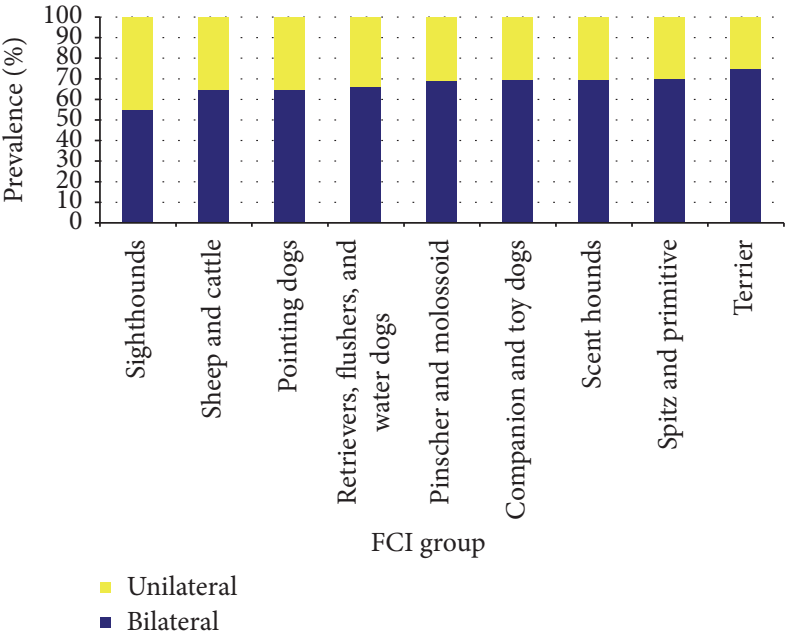

(b)

FIGURE 2: Unilateral and bilateral involvement in CHD. (a) By AKC group. (b) By FCI group.

in the data set (Supplemental Table 3) were also performed. Here again, similar findings are as seen for individual AKC and FCI groups. The detailed ORs of CHD for all dogs with $n>1000$ as well as all dogs with $n>100$ and a CHD prevalence of $>15 \%$ (the median value) are given in Supplemental Table 4.

3.4. Severity and Laterality of CHD. For those dogs with CHD, severity of the CHD was analyzed (Table 4). Severe CHD (score of 7) was more common in those with bilateral involvement, AKC groups of herding and working dogs, FCI groups of pinscher and sheep/cattle dogs, those living in the most southern latitudes $\left(<30^{\circ} \mathrm{N}\right)$, and those born in spring. Males had a slightly higher proportion of severe CHD. Regarding unilateral or bilateral involvement, bilateral disease was most prevalent in terriers and least prevalent in hybrid dogs within AKC groups (Figure 2(a)); bilateral disease was most prevalent in terriers and least prevalent in sighthounds within FCI groups (Figure 2(b)).

\section{Discussion}

Limitations of this study need to be acknowledged. Although we used a very large data set, it may not give the true prevalence of CHD, since it only represents the data on those dogs whose radiographs were submitted to the OFA. This predisposes to selection bias as it is not a truly random sample of the canine population [17]. Determination of the "true" prevalence would require a prospective radiographic exam between 2 and 5 years of age of every dog consecutively born, with a population of at least 1 million. Obviously such a study is impossible to perform. The OFA data set is therefore likely the best that can be presently obtained in the North America with the possible exception of the PennHIP ${ }^{\mathrm{TM}}$.
With these limitations in mind, there are several important findings. CHD is slightly more common in females, but with a large variation, ranging from 3.36 times more frequent in female Polish Tatra Sheepdogs to 1.63 times more frequent in male Afghan Hounds (Supplemental Table 1). CHD prevalence varies by breed, which was again demonstrated in this study, ranging from $77.7 \%$ in the bulldog to $0 \%$ in the Italian Greyhound. Many breeds demonstrated a mild increase in risk for CHD when born in winter and spring. $\mathrm{CHD}$ was unilateral in $33 \%$ of all dogs with $\mathrm{CHC}$. Unilateral involvement was more common in herding/sporting dogs and they had lower hip dysplasia scores. Finally, a new finding is that the prevalence of $\mathrm{CHD}$ is more common in dogs living in more southern latitudes.

This study confirms the marked variability in CHD prevalence by breed. In France, the highest prevalence of CHD was in the Cane Corso (59.7\%) and the lowest in the Siberian Husky (3.9\%) [18]. In a national Veterinary Medical Database from the entire USA [19], the OR of CHD was 10.2 in the Kuvasz with mixed breed dogs being the reference group. In a more recent study using the Veterinary Medical Database [20] the highest prevalence of CHD was $17.16 \%$ in the Newfoundland and $0.12 \%$ in the Scottish Terrier. In USA veterinary teaching hospitals, the prevalence of $\mathrm{CHD}$ was highest in the Rottweiler (35.4\%) and lowest in the miniature schnauzer dogs (1.5\%) [21]. In a Norwegian study comprised of Newfoundland, Leonberger, Labrador Retriever, and Irish Wolfhounds $(n=501)$, the highest prevalence of CHD was in the Newfoundland and the lowest in the Irish Wolfhound (OR 0.22 that of the Newfoundland) [22]. In Turkey, a study of 484 dogs from 7 different breeds revealed the highest prevalence in Doberman Pinschers (70.6\%) and the lowest in Golden Retrievers (50\%); the prevalence in Doberman Pinschers in this study in North America was low at 5.1\%. 
TABLE 4: Severity of CHD by demographic parameters.

\begin{tabular}{|c|c|c|c|c|c|c|c|}
\hline \multirow{2}{*}{ Parameter } & \multicolumn{3}{|c|}{ CHD severity } & \multicolumn{3}{|c|}{$\%$ severity } & \multirow{2}{*}{$p$ valu } \\
\hline & Mild & Moderate & Severe & Mild & Moderate & Severe & \\
\hline Age $(m o s \pm 1 s d)$ & $31.4 \pm 8.4$ & $32.0 \pm 8.9$ & $32.5 \pm 9.3$ & - & - & - & $<10^{-6}$ \\
\hline \multicolumn{8}{|l|}{ Sex } \\
\hline Male & 55,390 & 31,452 & 5,264 & 60.14 & 34.15 & 5.72 & \multirow{2}{*}{$<10^{-6}$} \\
\hline Female & 30,931 & 16,519 & 2,740 & 61.63 & 32.91 & 5.46 & \\
\hline \multicolumn{8}{|l|}{ Laterality } \\
\hline Bilateral & 51,085 & 36,754 & 7,537 & 53.56 & 38.54 & 7.90 & \multirow{2}{*}{$<10^{-6}$} \\
\hline Unilateral & 35,236 & 11,217 & 465 & 75.10 & 23.91 & 0.99 & \\
\hline \multicolumn{8}{|l|}{ AKC group } \\
\hline Herding & 17,011 & 8,906 & 1,723 & 61.54 & 32.22 & 6.23 & \multirow{7}{*}{$<10^{-6}$} \\
\hline Hound & 1,329 & 427 & 52 & 73.51 & 23.62 & 2.88 & \\
\hline Working & 22,469 & 14,379 & 2,371 & 57.29 & 36.66 & 6.05 & \\
\hline Sporting & 36,994 & 20,498 & 3,232 & 60.92 & 33.76 & 5.32 & \\
\hline Nonsporting & 4,452 & 2,062 & 413 & 64.27 & 29.77 & 5.96 & \\
\hline Terrier & 1,757 & 605 & 60 & 72.54 & 24.98 & 2.48 & \\
\hline Toy & 1,622 & 800 & 105 & 64.19 & 31.66 & 4.16 & \\
\hline \multicolumn{8}{|l|}{ FCI group } \\
\hline Sheep and cattle & 17,387 & 9,106 & 1,763 & 61.53 & 32.23 & 6.24 & \multirow{9}{*}{$<10^{-6}$} \\
\hline $\begin{array}{l}\text { Pinscher schnauzer, molossoid, and } \\
\text { Swiss Mtn/cattle dog }\end{array}$ & 20,179 & 13,430 & 2,371 & 56.08 & 37.33 & 6.59 & \\
\hline Terrier & 1,563 & 552 & 53 & 72.09 & 25.46 & 2.44 & \\
\hline Spitz and primitive & 4,106 & 2,120 & 325 & 62.68 & 32.36 & 4.96 & \\
\hline Scent hounds & 1,142 & 511 & 74 & 66.13 & 29.59 & 4.28 & \\
\hline Pointing dogs & 4,981 & 2,496 & 290 & 64.13 & 32.14 & 3.73 & \\
\hline Retrievers, flushers, and water dogs & 32,742 & 18,344 & 2,976 & 60.56 & 33.93 & 5.50 & \\
\hline Companion and toy dogs & 3,710 & 1,178 & 126 & 73.99 & 23.49 & 2.51 & \\
\hline Sighthounds & 181 & 85 & 3 & 67.29 & 31.60 & 1.12 & \\
\hline \multicolumn{8}{|l|}{ Geographic group } \\
\hline$<30^{\circ} \mathrm{N}$ & 2,760 & 1,183 & 199 & 66.63 & 28.56 & 4.80 & \multirow{4}{*}{$<10^{-6}$} \\
\hline $30-39^{\circ} \mathrm{N}$ & 41,628 & 22,781 & 3,711 & 61.11 & 33.44 & 5.45 & \\
\hline $40-49^{\circ} \mathrm{N}$ & 36,358 & 20,619 & 3,498 & 60.12 & 34.10 & 5.78 & \\
\hline$\geq 50^{\circ} \mathrm{N}$ & 5,335 & 3,235 & 580 & 58.31 & 35.36 & 6.34 & \\
\hline \multicolumn{8}{|l|}{ Season of birth } \\
\hline Autumn & 19,795 & 10,644 & 1,722 & 61.55 & 33.10 & 5.35 & \multirow{4}{*}{$<10^{-6}$} \\
\hline Winter & 21,512 & 12,101 & 1,960 & 60.47 & 34.02 & 5.51 & \\
\hline Spring & 24,728 & 14,456 & 2,503 & 59.32 & 34.68 & 6.00 & \\
\hline Summer & 20,286 & 10,770 & 1,819 & 61.71 & 32.76 & 5.53 & \\
\hline
\end{tabular}

It must be remembered that many of these studies used a different grading system than the OFA scores; however, it still confirms marked variability within breeds within each study.

The quoted prevalence of CHD is frequently different between different studies for a particular breed. When comparing the data of Witsberger et al. [20] to ours, the prevalence of CHD for the Newfoundland was $17.2 \%$ versus $20.0 \%$, Saint Bernard $14.7 \%$ versus 36.8\%, Rottweiler $10.3 \%$ versus $12.5 \%$, German Shepherd 10.3\% versus 16.3\%, Golden Retriever $8.5 \%$ versus $14.9 \%$, Labrador Retriever 7.4\% versus 9.2\%, Bulldog 4.4\% versus 68.9\%, Doberman Pinscher $1.3 \%$ versus 5.1\%, and Greyhound $0.4 \%$ versus $2.1 \%$, respectively. This demonstrates that the sampling technique/composition of the data set markedly impacts the prevalence value as previously mentioned. Prevalence amongst each breed within a country, or region, is likely a result of gene flow, bottle necks, popular sire effects, and the efforts of individuals and breed clubs to impact the prevalence and severity of CHD in a particular breed.

We noted a slight increase in CHD in females with marked differences by breed. Several studies noted no sex difference in the prevalence of CHD. In Norway, Turkey, and 
the United Kingdom no sex differences were noted for the various breeds studied [22-25]. In Sweden, CHD was 1.14 times more common in female German Shepherds compared to males [26]. In the United States, sex differences were noted in Golden Retrievers [27]; the prevalence of $\mathrm{CHD}$ was $5.1 \%$ in intact males, $10.3 \%$ in males neutered early, $0 \%$ in males neutered late, $39 \%$ in intact females, $4.5 \%$ in females neutered early, and $0 \%$ in females neutered late. The status of neutering in the OFA registry is not given, so we cannot compare our findings to those of Torres de la Riva [27].

The prevalence of unilateral CHD was $33 \%$ in this study. The prevalence of unilateral CHD was 35\% in a New York study of 1022 dogs consisting of Labrador Retrievers, Golden Retrievers, German Shepherds, and crossbreeds [2]. In Pennsylvania, it was 6\% in 133 Greyhounds. A recent study of multiple breeds from Italy noted an overall percentage of unilateral CHD of $31.5 \%$ [28], strikingly similar to the $33 \%$ in this study and the $35 \%$ of Lust et al. [2]. This is the first study to investigate the proportion of unilateral $\mathrm{CHD}$ by AKC/FCI groups; for AKC groups it was highest in herding dogs (35.4\%) and lowest in terriers (27.5\%); for FCI groups it was highest in sheep/cattle dogs (35.4\%) and lowest in terriers (25.1\%) (Table 1).

Few studies discuss season of birth and CHD. In Norway [29], the OR for CHD (Newfoundland, Leonberger, Labrador Retriever, and Irish Wolfhounds) was 3.94 times higher in autumn and 1.85 times higher in winter compared to spring. In another Norwegian study [9], pointers had an increase in CHD in those born in August to February, Labrador Retrievers September to February, with no seasonal effect on CHD in German Shepherds or Golden Retrievers. In Finland [7], German Shepherds born in spring or summer had less CHD. In England [10], Labrador Retrievers and Gordon Setters had less CHD when born in July through October. In New Zealand [8], Labrador Retrievers and Rottweilers had less CHD when born in autumn, but no seasonal variation was observed for German Shepherds or Golden Retrievers. In aggregate, the previous studies in the Northern Hemisphere noted that dogs born in autumn and/or winter months demonstrate a higher prevalence of CHD. In this study we noted an increase of CHD primarily in winter and spring months. When reviewing the data from Supplemental Table $3,563,403$ of the 619,825 dogs $(81.4 \%)$ showed a seasonal variation. Of these 536,403, 313,202 (55.6\%) had the highest percentage in winter, $229,925(40.8 \%)$ in spring, and 20,276 (3.6\%) in autumn.

There are several postulated reasons for seasonal differences in CHD. One is the relationship between hip muscle development and season. The most critical time for canine hip joint development is between 3 and 9 months of age $[8,30]$; cage confinement during this crucial period has a protective effect on the hip [30]. The proposed explanation is that puppies born in winter spend more time in cages/indoors than in free activities, and indoor confinement may keep the hips in flexion and abduction lessening the development of CHD [29]. The same has been noted in human DDH, where carrying the infant in positions of hip abduction and flexion reduces the incidence of DDH [31-35] while swaddling in extension increases the incidence of DDH $[5,36,37]$. Our results refute a winter protective effect in $\mathrm{CHD}$. A second explanation is that puppies born in late autumn or early winter, compared to those born in spring or early summer, do not get as much physical exercise. Puppies getting less physical exercise may develop weaker hip musculature than those with a lot of outdoor activity, which when combined with rapid skeletal growth results in weakened constraints on the hip, subsequent subluxation, and $\mathrm{CHD}[8,22,29,30]$. This can explain the increase in CHD in dogs born in late autumn/early winter and corroborates the findings from New Zealand, England, and our study, while conflicting with the data from Norway, Finland, and Sweden.

Another postulated mechanism for CHD seasonal variation is diet and weight gain in puppies. Dogs with limited weight gain in early life have a lower prevalence of $\mathrm{CHD}$ $[2,22,29,38,39]$. In cold winter months dogs have increased food intake [40, 41], and if not accompanied by an increase in energy consumption (e.g., activity), the dog will gain weight. Increased body weight increases the stress across the developing hip joint leading to subluxation [17, 42, 43]. Vitamin $\mathrm{D}$ plays a role in $\mathrm{DDH}$, as humans with homozygosity for the mutant Taq1 vitamin $\mathrm{D}$ receptor $t$ allele demonstrate increased acetabular dysplasia [44]. Vitamin D levels may vary by season due to seasonal variation in vitamin $\mathrm{D}$ dietary content in both humans and animals [45-52]. Low vitamin D levels and increased body fat in winter may result in more CHD. Finally, various dietary factors differ by season and could result in seasonal differences in hormones in milk (vitamin D, relaxin, and vitamin C) and secondarily influence hip development [52-57].

This is the first description of an increased prevalence of CHD in more southern latitudes. This was true even when multivariate regression logistic analysis was performed adjusting for breed group, gender, and season of birth. One potential explanation is that the generally warmer climate in more southern latitudes may result in a general increase in physical activity at all times, with the hips being less abducted and flexed, resulting in more CHD. Another potential explanation is that the gene pools may be different in different latitudes. Finally, other environmental factors such as diet as discussed above may be involved, resulting in increased CHD. Perhaps the dogs in the more southern latitudes are heavier and place more stress across the hip. It could also be that the dogs in the warmer more southern latitudes grow more rapidly early in life, which is a well-known contributing factor to $\mathrm{CHD}[38,39]$. This finding and potential explanations will require further study.

There are marked differences and similarities between $\mathrm{DDH}$ and $\mathrm{CHD}$ (Table 5). The most striking is the difference in incidence/prevalence by race/breed. Prevalence/incidence variation in humans is higher (950-fold difference in Native Americans compared to Africans in Africa) than canines (96-fold difference in the bulldog compared to the whippet) (Supplemental Table 1). DDH occurs predominantly in females (75\%) for all races [5], while for CHD the prevalence was only slightly higher in females compared to males (Table 1). However there are large sex variations in CHD which ranged from 3.4 times more frequent in female Polish Tatra Sheepdogs to 1.6 times more frequent 


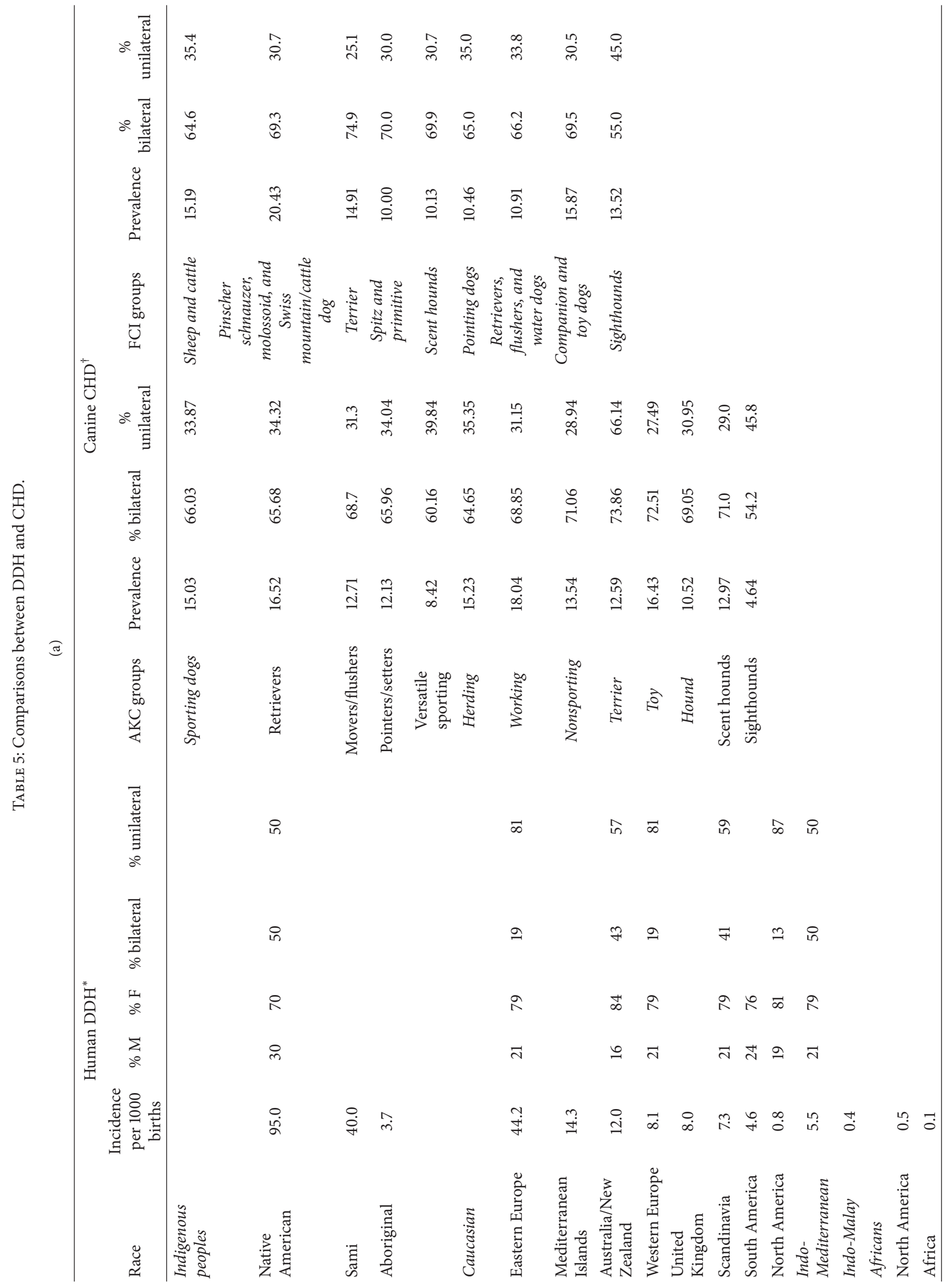




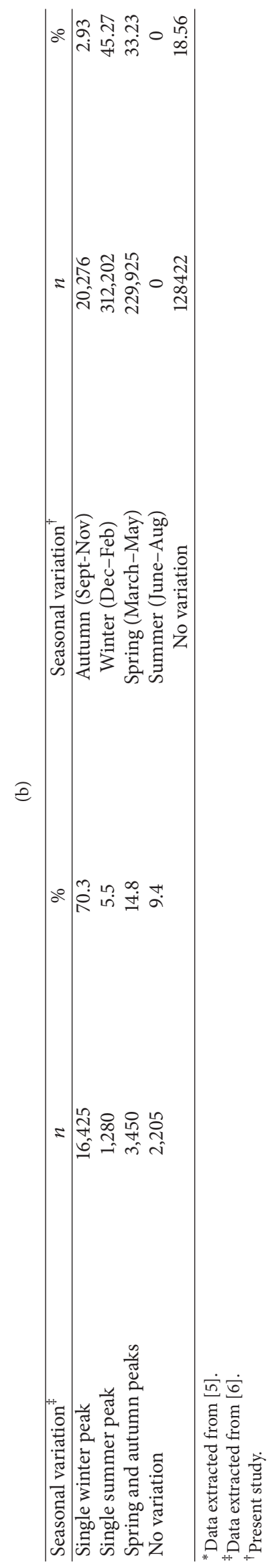


in male Afghan Hounds. DDH is usually unilateral (63.4\%) [5] compared to CHD which is usually bilateral (67\%). DDH demonstrates a seasonal variation in $\sim 91.0 \%$ of cases [6], and $81.4 \%$ in CHD, which is remarkably similar. DDH was most prevalent when the baby was born in winter months (70.3\%); CHD was most prevalent when the puppy was born in winter and spring. $\mathrm{DDH}$ is more common in northern latitudes, while CHD is more common in southern latitudes $[5,6]$. This latitudinal difference has also been noted in children with Perthes' disease [58]. Within DDH there are two different types, the typical infantile DDH and the late onset adolescent/adult acetabular dysplasia [59]. The older group, when compared to the infantile group, demonstrated a lower female predominance (88.0 versus $98.0 \%$ ) with more bilateral involvement (61.2\% versus $45.1 \%)$. Our findings in $\mathrm{CHD}$ more closely mirror the demographics of $\mathrm{DDH}$ in the late onset group.

In conclusion, the prevalence of CHD differed markedly by breed, having a slight female predominance but with significant variability by breed, was unilateral in about onethird of cases, and often demonstrated a seasonal variation with a mild increase when the dog was born in spring and winter months. Most interestingly, $\mathrm{CHD}$ was more prevalent in the more southern latitudes. This information is important to owners/breeders, suggesting that monitoring of puppies for signs of CHD should be undertaken during the birth months when there is an increased OR of CHD for those affected breeds and/or AKC groups, especially in more southern latitudes. The similarities between $\mathrm{CHD}$ and $\mathrm{DDH}$ are striking, especially late onset $\mathrm{DDH}$, and suggest that comparative studies of both disorders should lead to a better understanding of a problem that leads to debilitating hip osteoarthritis in both canines and humans.

\section{Conflicts of Interest}

The authors declare that they have no conflicts of interest.

\section{Acknowledgments}

The authors wish to thank Mr. Eddi Dzuik and Jon Curby, Orthopaedic Foundation for Animals, for granting the authors access to the entire hip dysplasia registry. This research was supported in part by the Garceau Professorship Endowment, Indiana University, School of Medicine, Department of Orthopaedic Surgery, and the Rapp Pediatric Orthopaedic Research Endowment, Riley Children's Foundation, Indianapolis, Indiana.

\section{References}

[1] W. H. Riser, "Canine hip dysplasia: cause and control," Journal of the American Veterinary Medical Association, vol. 165, no. 4, pp. 360-362, 1974.

[2] G. Lust, J. C. Geary, and B. E. Sheffy, "Development of hip dysplasia in dogs," American Journal of Veterinary Research, vol. 34, no. 1, pp. 87-91, 1973.
[3] G. Lust, V. T. Rendano, and B. A. Summers, "Canine hip dysplasia: concepts and diagnosis., Journal of the American Veterinary Medical Association, vol. 187, no. 6, pp. 638-640, 1985.

[4] G. Lust, A. J. Williams, N. Burton-Wurster et al., "Joint laxity and its association with hip dysplasia in Labrador retrievers," American Journal of Veterinary Research, vol. 54, no. 12, pp. 1990-1999, 1993.

[5] R. T. Loder and E. N. Skopelja, "The epidemiology and demographics of hip dysplasia," ISRN Orthopedics, vol. 2011, Article ID 238607, 46 pages, 2011.

[6] R. T. Loder and C. Shafer, "Seasonal variation in children with developmental dysplasia of the hip," Journal of Children's Orthopaedics, vol. 8, no. 1, pp. 11-22, 2014.

[7] M. Leppänen, K. Mäki, J. Juga, and H. Saloniemi, "Factors affecting hip dysplasia in German shepherd dogs in Finland: efficacy of the current improvement programme," Journal of Small Animal Practice, vol. 41, no. 1, pp. 19-23, 2000.

[8] A. J. Worth, J. P. Bridges, N. J. Cave, and G. Jones, "Seasonal variation in the hip score of dogs as assessed by the New Zealand Veterinary Association Hip Dysplasia scheme," New Zealand Veterinary Journal, vol. 60, no. 2, pp. 110-114, 2012.

[9] I. Hanssen, "Hip dysplasia in dogs in relation to their month of birth," Veterinary Record, vol. 128, no. 18, pp. 425-426, 1991.

[10] J. L. N. Wood and K. H. Lakhani, "Effect of month of birth on hip dysplasia in labrador retrievers and Gordon setters," Veterinary Record, vol. 152, no. 3, pp. 69-72, 2003.

[11] S. Ohlerth, J. Lang, A. Busato, and C. Gaillard, "Estimation of genetic population variables for six radiographic criteria of hip dysplasia in a colony of Labrador Retrievers," American Journal of Veterinary Research, vol. 62, no. 6, pp. 846-852, 2001.

[12] S. Malm, W. F. Fikse, B. Danell, and E. Strandberg, "Genetic variation and genetic trends in hip and elbow dysplasia in Swedish Rottweiler and Bernese Mountain Dog," Journal of Animal Breeding and Genetics, vol. 125, no. 6, pp. 403-412, 2008.

[13] D. C. Coile, Barron's Encyclopedia of Dog Breeds, Barron's Educational Series, Hauppage, New York, NY, USA, 2nd edition, 2005.

[14] FCI breeds nomenclature, http://www.fci.be/en/Nomenclature/.

[15] H. G. Parker, "Genomic analyses of modern dog breeds," Mammalian Genome, vol. 23, no. 1, pp. 19-27, 2012.

[16] M. Rimbault and E. A. Ostrander, "So many doggone traits: mapping genetics of multiple phenotypes in the domestic dog," Human Molecular Genetics, vol. 21, no. 1, pp. R52-R57, 2012.

[17] F. H. Comhaire and F. Snaps, "Comparison of two canine registry databases on the prevalence of hip dysplasia by breed and the relationship of dysplasia with body weight and height," American Journal of Veterinary Research, vol. 69, no. 3, pp. 330333, 2008.

[18] J.-P. Genevois, D. Remy, E. Viguier et al., "Prevalence of hip dysplasia according to official radiographic screening, among 31 breeds of dogs in France. A retrospective study, Veterinary and Comparative Orthopaedics and Traumatology, vol. 21, no. 1, pp. 21-24, 2008.

[19] E. LaFond, G. J. Breur, and C. C. Austin, "Breed susceptibility for developmental orthopedic diseases in dogs," Journal of the American Animal Hospital Association, vol. 38, no. 5, pp. 467477, 2002.

[20] T. H. Witsberger, J. Armando Villamil, L. G. Schultz, A. W. Hahn, and J. L. Cook, "Prevalence of and risk factors for hip dysplasia and cranial cruciate ligament deficiency in dogs," Journal of the American Veterinary Medical Association, vol. 232, no. 12, pp. 1818-1824, 2008. 
[21] J. L. Rettenmaier, G. G. Keller, J. C. Lattimer, E. A. Corley, and M. R. Ellersieck, "Prevalence of canine hip dysplasia in a veterinary teaching hospital population," Veterinary Radiology and Ultrasound, vol. 43, no. 4, pp. 313-318, 2002.

[22] R. I. Krontveit, A. Nødtvedt, B. K. Sævik, E. Ropstad, H. K. Skogmo, and C. Trangerud, "A prospective study on Canine Hip Dysplasia and growth in a cohort of four large breeds in Norway (1998-2001)," Preventive Veterinary Medicine, vol. 97, no. 3-4, pp. 252-263, 2010.

[23] M. Sarierler, "Comparison of femoral inclination angle measurements in dysplastic and nondysplastic dogs of different breeds," Acta Veterinaria Hungarica, vol. 52, no. 2, pp. 245-252, 2004.

[24] J. L. N. Wood, K. H. Lakhani, and R. Dennis, "Heritability and epidemiology of canine hip-dysplasia score in flat-coated retrievers and Newfoundlands in the United Kingdom," Preventive Veterinary Medicine, vol. 46, no. 2, pp. 75-86, 2000.

[25] B. Freeman, V. B. Evans, and N. R. McEwan, "Canine hip dysplasia in Irish water spaniels: two decades of gradual improvement," Veterinary Record, vol. 173, no. 3, pp. 72-73, 2013.

[26] A. Hedhammar, S. E. Olsson, S. A. Andersson et al., "Canine hip dysplasia: study of heritability in 401 litters of German Shepherd dogs," Journal of the American Veterinary Medical Association, vol. 174, no. 9, pp. 1012-1016, 1979.

[27] G. Torres de la Riva, B. L. Hart, T. B. Farver et al., "Neutering dogs: effects on joint disorders and cancers in Golden Retrievers," PLoS ONE, vol. 8, no. 2, Article ID e55937, 2013.

[28] S. Citi, M. Vignoli, M. Modenato, F. Rossi, and J. P. Morgan, "A radiological study of the incidence of unilateral canine hip dysplasia," Schweizer Archiv fur Tierheilkunde, vol. 147, no. 4, pp. 173-178, 2005.

[29] R. I. Krontveit, A. Nødtvedt, B. K. Sævik, E. Ropstad, and C. Trangerud, "Housing- and exercise-related risk factors associated with the development of hip Dysplasia as determined by radiographic evaluation in a prospective cohort of Newfoundlands, Labrador retrievers, Leonbergers, and Irish wolfhounds in Norway," American Journal of Veterinary Research, vol. 73, no. 6, pp. 838-846, 2012.

[30] W. H. Riser, "A new look at developmental subluxation and dislocation: hip dysplasia in the dog," Journal of Small Animal Practice, vol. 4, no. 6, pp. 421-434, 1963.

[31] M. Janecek, "Congenital hip dislocation in children in Northern Korea," Acta chirurgiae orthopaedicae et traumatologiae Cechoslovaca, vol. 23, no. 1, pp. 2-5, 1956.

[32] J. R. Corea, "Is congenital dislocation of the hip rare in Sri Lanka?" The Ceylon Medical Journal, vol. 37, no. 3, p. 96, 1992.

[33] J. Edelstein, "Congenital dislocation of the hip in the Bantu," The Journal of Bone \& Joint Surgery-British Volume, vol. 48, no. 2, p. $397,1966$.

[34] J. C. Griffiths, "Dislocated hip in East African infants and children," Postgraduate Medical Journal, vol. 46, no. 532, pp. 8691, 1970.

[35] A. P. Skirving and W. J. Scadden, "The African neonatal hip and its immunity from congenital dislocation," Journal of Bone and Joint Surgery - Series B, vol. 61, no. 3, pp. 339-341, 1979.

[36] S. H. Blatt, “To swaddle, or not to swaddle? Paleoepidemiology of developmental dysplasia of the hip and the swaddling dilemma among the indigenous populations of North America," American Journal of Human Biology, vol. 27, no. 1, pp. 116-128, 2015.
[37] R. B. Salter, "Etiology, pathogenesis and possible prevention of congenital dislocation of the hip," Canadian Medical Association Journal, vol. 98, no. 20, pp. 933-945, 1968.

[38] R. D. Kealy, S. E. Olsson, K. L. Monti et al., "Effects of limited food consumption on the incidence of hip dysplasia in growing dogs," Journal of the American Veterinary Medical Association, vol. 201, no. 6, pp. 857-863, 1992.

[39] R. D. Kealy, D. F. Lawler, J. M. Ballam et al., "Evaluation of the effect of limited food consumption on radiographic evidence of osteoarthritis in dogs," Journal of the American Veterinary Medical Association, vol. 217, no. 11, pp. 1678-1680, 2000.

[40] M. D. Finke, "Evaluation of the energy requirements of adult kennel dogs," Journal of Nutrition, vol. 121, no. 11, pp. S22-S28, 1991.

[41] J. L. Durrer and J. P. Hannon, "Seasonal variations in caloric intake of dogs living in an arctic environment," The American Journal of Physiology, vol. 202, no. 2, pp. 375-378, 1962.

[42] S. L. Sanderson, "The epidemic of canine obesity and its role in osteoarthritis," Israel Journal of Veterinary Medicine, vol. 67, no. 4, pp. 195-202, 2012.

[43] A. J. German, "The growing problem of obesity in dogs and cats," Journal of Nutrition, vol. 136, no. 7, pp. 1940S-1946S, 2006.

[44] B. Kapoor, C. Dunlop, C. Wynn-Jones, A. A. Fryer, R. C. Strange, and N. Maffulli, "Vitamin D and oestrogen receptor polymorphisms in developmental dysplasia of the hip and primary protrusio acetabuli-a preliminary study," Journal of Negative Results in BioMedicine, vol. 6, article 7, 2007.

[45] R. P. Stryd, T. J. Gilbertson, and M. N. Brunden, "A seasonal variation study of 25-hydroxyvitamin D3 serum levels in normal humans," Journal of Clinical Endocrinology and Metabolism, vol. 48, no. 5, pp. 771-775, 1979.

[46] M. J. McKenna, "Differences in vitamin D status between countries in young adults and the elderly," The American Journal of Medicine, vol. 93, no. 1, pp. 69-77, 1992.

[47] S. S. Harris and B. Dawson-Hughes, "Seasonal changes in plasma 25-hydroxyvitamin D concentrations of young American black and white women," American Journal of Clinical Nutrition, vol. 67, no. 6, pp. 1232-1236, 1998.

[48] R. Andersen, C. Mølgaard, L. T. Skovgaard et al., “Teenage girls and elderly women living in northern europe have low winter vitamin D status," European Journal of Clinical Nutrition, vol. 59, no. 4, pp. 533-541, 2005.

[49] C. Karohl, S. Su, M. Kumari et al., "Heritability and seasonal variability of vitamin D concentrations in male twins," American Journal of Clinical Nutrition, vol. 92, no. 6, pp. 1393-1398, 2010.

[50] G. Snellman, H. Melhus, R. Gedeborg et al., "Seasonal genetic influence on serum 25-hydroxyvitamin D levels: a twin study," PLoS ONE, vol. 4, no. 11, 2009.

[51] M. Wacker and M. F. Holiack, "Vitamin D-effects on skeletal and extraskeletal health and the need for supplementation," Nutrients, vol. 5, no. 1, pp. 111-148, 2013.

[52] J. E. Parker, K. I. Timm, B. B. Smith et al., "Seasonal interaction of serum vitamin D concentration and bone density in alpacas," American Journal of Veterinary Research, vol. 63, no. 7, pp. 948953, 2002.

[53] C. J. Laing, R. Malik, D. I. Wigney, and D. R. Fraser, "Seasonal vitamin D status of Greyhounds in Sydney," Australian Veterinary Journal, vol. 77, no. 1, pp. 35-38, 1999.

[54] P. H. Mäenpää, T. Koskinen, and E. Koskinen, "Serum profiles of vitamins $\mathrm{A}, \mathrm{E}$ and $\mathrm{D}$ in mares and foals during different 
seasons," Journal of animal science, vol. 66, no. 6, pp. 1418-1423, 1988.

[55] Y. Adkins, A. J. Lepine, and B. Lönnerdal, "Changes in protein and nutrient composition of milk throughout lactation in dogs," American Journal of Veterinary Research, vol. 62, no. 8, pp. 12661272, 2001.

[56] L. T. Goldsmith, G. Lust, and B. G. Steinetz, "Transmission of relaxin from lactating bitches to their offspring via suckling," Biology of Reproduction, vol. 50, no. 2, pp. 258-265, 1994.

[57] C. R. Heinze, L. M. Freeman, C. R. Martin, M. L. Power, and A. J. Fascetti, "Comparison of the nutrient composition of commercial dog milk replacers with that of dog milk," Journal of the American Veterinary Medical Association, vol. 244, no. 12, pp. 1413-1422, 2014.

[58] D. C. Perry, D. M. G. MacHin, D. Pope et al., "Racial and geographic factors in the incidence of Legg-Calvé-Perthes' disease: a systematic review," American Journal of Epidemiology, vol. 175, no. 3, pp. 159-166, 2012.

[59] C. B. Lee, A. Mata-Fink, M. B. Millis, and Y.-J. Kim, "Demographic differences in adolescent-diagnosed and adultdiagnosed acetabular dysplasia compared with infantile developmental dysplasia of the hip," Journal of Pediatric Orthopaedics, vol. 33, no. 2, pp. 107-111, 2013. 

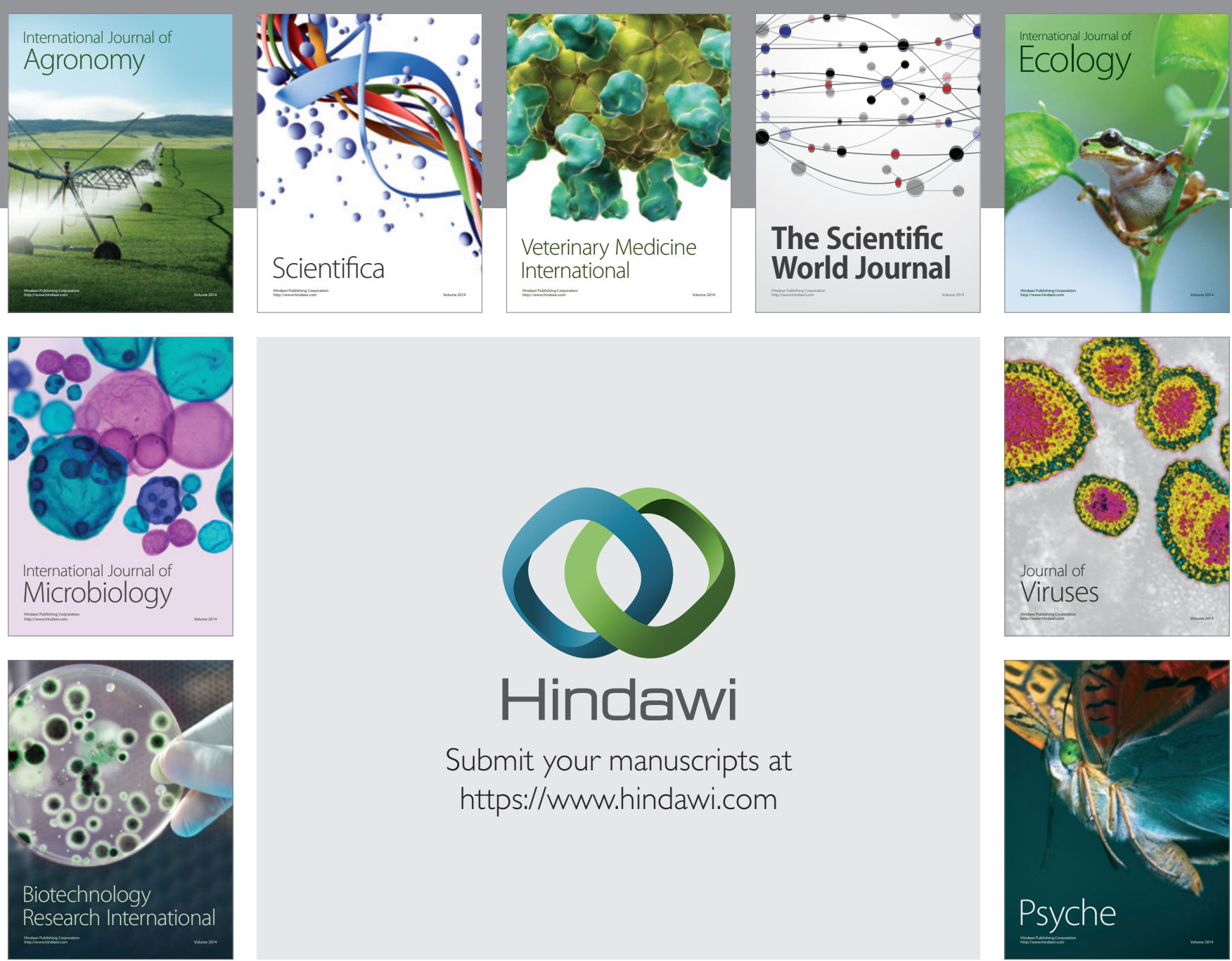

\section{Hindawi}

Submit your manuscripts at

https://www.hindawi.com
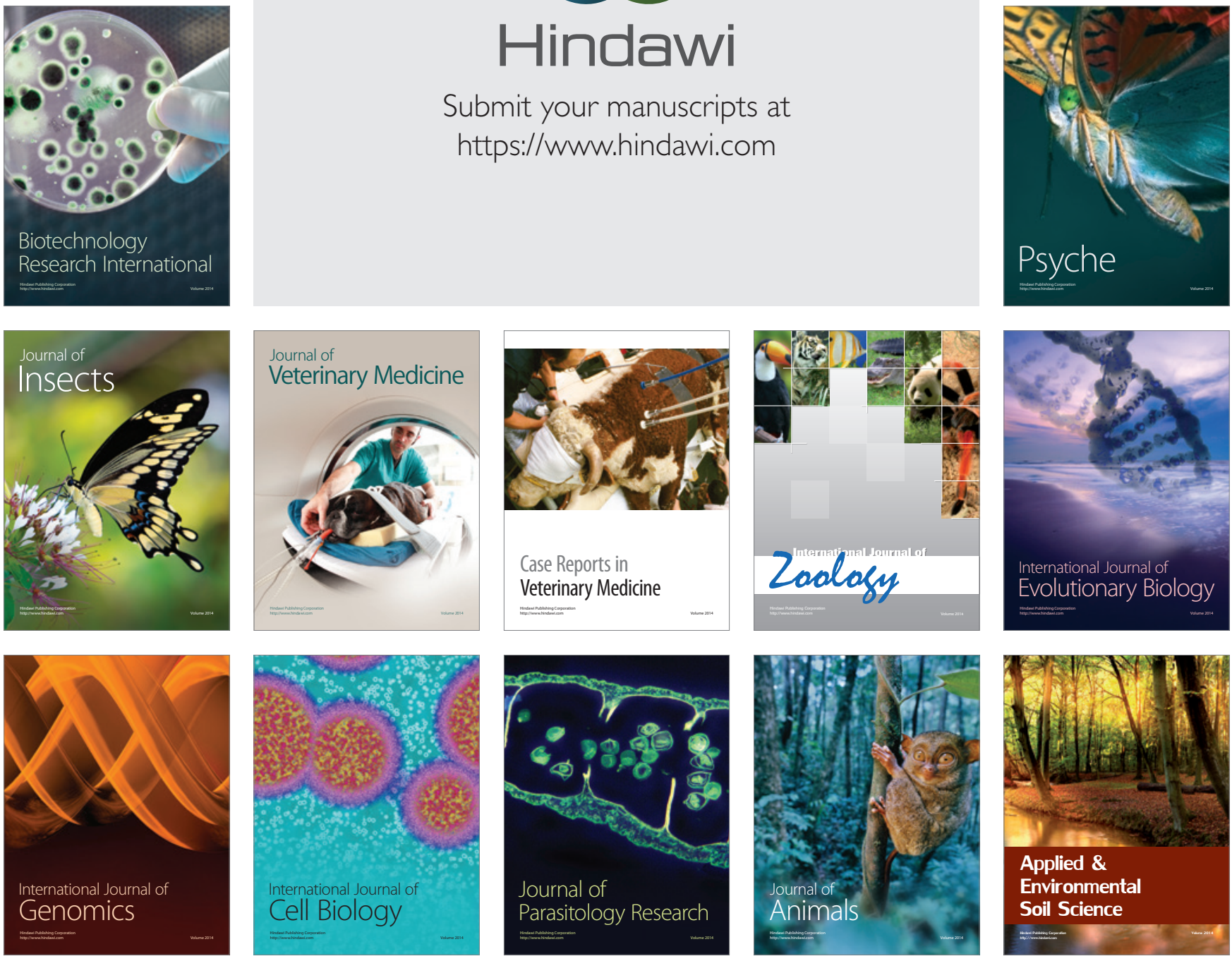